\title{
PA-095 A CROSS-SECTIONAL STUDY OF HEPATITIS B VIRUS INFECTION IN HIV-INFECTED CHILDREN IN WINDHOEK, NAMIBIA
}

Cynthia Tamandjou, ${ }^{1}$ Francina Kaindjee-Tjituka, ${ }^{2}$ Laura Brandt, ${ }^{3}$ Mark Cotton, ${ }^{3}$ Etienne Nel, ${ }^{1}$ Wolfgang Preiser, ${ }^{1}$ Monique Andersson'. ${ }^{1}$ Stellenbosch University, South Africa; ${ }^{2}$ Ministry of Health and Social Services, Namibia; ${ }^{3}$ I-TECH, Namibia

10.1136/bmjgh-2016-000260.126

Background Hepatitis B virus (HBV) remains endemic in Africa and an important co-morbidity in the HIV epidemic. The HIV treatment guidelines of the World Health Organisation (WHO) recommend tenofovir-lamivudine (or emtricitabine) as first-line therapy for HIV-HBV co-infection management in children $\geq 10$ years old. However, many children in sub-Saharan Africa are not screened for HBV and may remain on lamivudine monotherapy for many years. This study aimed to characterise HBV infection in HIV-infected children in Namibia.

Methods The study included HIV-infected/HBsAg-positive children, exposed to lamivudine monotherapy, attending the Katutura paediatric HIV clinic in Windhoek, Namibia. Dried blood spots and serum samples were collected from participants. Serological investigations were performed using Murex assays. HBV DNA viral load was determined using the automated AmpliPrep/COBAS TaqMan HBV test V2.0. Genotyping and mutation analysis were performed through the NCBI HBV Genotyping tool (www.ncbi.nlm.nih.gov/ projects/genotyping/ formpage.cgi) and Geno2Pheno (http:// hbv.geno2pheno.org/ index.php).

Results To date, 14 children have been enrolled; of whom 14 DBS and 11 serum samples were analysed. HBsAg was detected in 10 children (90\%; 10/11); 7 were HBeAg-positive/ $\mathrm{HBeAb}$-negative and $3 \mathrm{HBeAg}$-negative. Among HBeAg-negatives, 1 was $\mathrm{HBeAb}$-negative and 2 were $\mathrm{HBeAb}$-positive. One child was non-reactive for all markers. Of the 14 children, 7(50\%) tested HBV DNA-positive. Lamivudine drug-associated resistance variants, together with immune escape mutants in the overlapping surface gene, were identified in these children. Resistance mutation patterns included: $r t \mathrm{~V} 173 \mathrm{~L}+r t \mathrm{~L} 180 \mathrm{M}+r t-\mathrm{M} 204 \mathrm{~V}$ (4/7; 57\%), $r t \mathrm{~L} 80 \mathrm{I}+r t \mathrm{~V} 173 \mathrm{~L}+r t \mathrm{~L} 180 \mathrm{M}+r t \mathrm{M} 204 \mathrm{I}(1 / 7 ; 14 \%)$ and $r t \mathrm{~L} 180 \mathrm{M}$ 
$+r t \mathrm{M} 204 \mathrm{~V}$ (2/7; 29\%); with the overlapping sE164D and/or sI195M variants. HBV strains belonged to genotype E $(6 / 7,86 \%)$ and genotype D3 (1/7, 14\%).

Conclusions Half of the children included in this study had detectable HBV DNA and showed lamivudine resistance. Uncontrolled HBV infection is associated with an increased risk of severe liver damage and hepatocellular carcinoma. HBsAg screening of HIV-infected children, using cost-effective point-of-care methods, and treatment with tenofovir should be made more widely available in resource-limited settings. 